Methods Explant-derived HPASMCs from unaffected donors or PAH patients with identified BMPR-II mutations were studied. The transcriptional responses of ID1, ID2, PAI1 and CTGF to BMP4, TGF $\beta 1$ or co-treatment were examined by qPCR In some experiments, cells were pre-incubated with cycloheximide or pharmacological inhibitors of ALK5 (SD208), MAP kinase (U0126), TAK1 or p38 MAP kinase (SB203580). The roles of Smad2, Smad3, Smad6 and Smad7 were investigated using siRNAs. Smad-dependent transcription was examined using the BMP-responsive luciferase reporter (BRE-luc) and the TGF-responsive luciferase reporter, CAGA12-luc. Protein lysates collected at 1 and 4 hrs were immunoblotted for phosphorylated and total Smads and candidate kinases. In some experiments, nuclear and cytoplasmic fractions were prepared and immunoblotted.

Results BMP4 induced ID1 and ID2 expression at 1, 4 and 24h whereas TGF $\beta 1$ induced ID1/2 at $1 \mathrm{~h}$ and repressed them at $4 \mathrm{~h}$ and 24h. TGF $\beta 1$, but not BMP4, induced PAI1 and CTGF expression. BMP4 did not alter TGF $\beta 1$-mediated transcriptional responses. In contrast, TGF $\beta 1$ attenuated BMP4-mediated ID1/2 induction and the BRE-luc response in donor HPASMCs. Moreover, TGF $\beta 1$ abolished BMP4 responses in PAH PASMCs with BMPR-II mutations. This was reversed by the ALK5 inhibitor, SD208, but not by cycloheximide or a TAK1 inhibitor. BMP-Smad phosphorylation and nuclear translocation did not differ between co-treatment and treatment with individual ligands. Smad3 siRNA, but not Smad2, Smad6 or Smad7, reversed the inhibitory effect of TGF $\beta 1$.

Conclusions TGF $\beta 1$ negatively regulates BMP4-mediated ID genes transcription by interfering with BMP-Smad signalling via Smad3, abolishing the BMP signals in cells with BMPR-II mutations. These data reinforce the notion that TGF $\beta 1$ plays a pathogenic role in $\mathrm{PAH}$, via direct inhibition of the attenuated $\mathrm{BMP}$ response caused by BMPR-II insufficiency.

\section{S39 WHOLE EXOME SEQUENCING IN CHRONIC THROMBOEMBOLIC PULMONARY HYPERTENSION REVEALS BIOLOGICALLY PLAUSIBLE NOVEL GENETIC VARIATION}

doi:10.1136/thoraxjnl-2012-202678.045

C McCabe, K Sheares, S Graf, J Pepke-Zaba, N Morrell. Papworth Hospital, Cambridge, United Kingdom

Chronic thromboembolic pulmonary hypertension (CTEPH) is a disease with no known heritability, the cumulative incidence of which has been placed at between 0.5 and $9.1 \%$ following acute pulmonary embolism [1,2]. A majority consensus on its development has emerged predicated on the theory of disordered thrombus resolution following venous thromboembolism. This in part stems from epidemiological data but is also based on studies implicating established clinical and laboratory factors, a central theorem being that individuals respond differently to acute thrombotic insult.

Given traditional prothrombotic factors explain less than $10 \%$ of reported cases of CTEPH, we hypothesised a significant burden of disease is accounted for by unidentified genetic factors. Using an unbiased approach of exome capture, we selected 20, deeply phenotyped, young individuals (11 female) who had suffered large PE that subsequently developed haemodynamically confirmed CTEPH despite anticoagulation. Individuals with known prothrombotic tendency and significant comorbidity were excluded. Selected patients were White Caucasian origin.

Following ethical approval, DNA was extracted from 20 whole blood samples and libraries prepared. Whole exome sequencing was undertaken using Agilent's Sure Select Exome capture kit on the Hiseq 2000 platform prior to bioinformatic analysis. 75 bp paired-end reads were aligned to the $\mathrm{H} 19$ Reference genome and the pipeline annotated for SNPs and indels. Intergenic, intronic and UTR variants were removed from the dataset with minimum mapping quality score and 20x read-depth coverage stipulated for variant calls.

Consecutive filtres were applied, firstly retaining those predicted to be pathogenic flagged as splice-site, essential splice-site, missense, frameshift-coding, non-synonymous coding, NMD-transcript, within-mature-miRNA, STOP-gained and STOP-lost. Variants also present in twenty locally sourced normal controls were discarded. Additional filtres were subsequently applied to exclude variants already listed in dbSNP/1000 genomes data with remaining variants split into heterozygous and homozygous groups (Table 1). Our final candidate list contains novel DNA variants lying within pathways known to mediate both inflammation and endothelial dysfunction in the pulmonary vasculature. Detailed downstream biological analysis of our variant effects following validation measures may lead to the uncovering of novel mechanisms in this difficult disease.

\section{References}

1. Surie S, Gibson NS, Gerdes VE, Bouma BJ, van Eck-Smit $\mathrm{BL}$, Buller HR, Bresser P. Active search for chronic thromboembolic pulmonary hypertension does not appear indicated after acute pulmonary embolism. Thromb Res. 2010; 125: 202-5

2. Becattini C, Agnelli G, Pesavento R, Silingardi M, Poggio R, Taliani MR, Ageno W. Incidence of chronic thromboembolic pulmonary hypertension after a first episode of pulmonary embolism. Chest. 2006; 130:172-5.

Abstract S39 Table 1

\begin{tabular}{|l|l|l|l|l||}
\hline N=20 & Heterozygous & & Homozygous & \\
\hline (>5samples) & SNP & Gene & SNP & Gene \\
\hline Pathogenic & $33260(13767)$ & $11009(6267)$ & $1251(423)$ & $587(112)$ \\
\hline $\begin{array}{l}\text { Novel } \\
\text { (dbSNPI } \\
\text { genomes }\end{array}$ & $6662(332)$ & $5868(268)$ & $447(70)$ & $312(65)$ \\
\hline $\begin{array}{l}\text { Not in } \\
\text { Normal } \\
\text { Controls }\end{array}$ & $5002(43)$ & $3741(57)$ & $241(10)$ & $189(10)$ \\
\hline
\end{tabular}

Table 1 shows the number of SNPs and genes containing those SNPs for both heterozygous and homozygous variants following each filtering sep. Bracketed values show the number of $S N P$ sigenes with those SNPs present in 5 or more samples. 